\title{
Differential responses of human articular cartilage to retinol
}

\author{
RONALD W. JUBB \\ From Strangeways Research Laboratory, Worts' Causeway, Cambridge
}

SUMmARY An in-vitro study has been made of the response of aged human articular cartilage to the catabolic agent retinol. Weight bearing cartilage from the femoral condyle degrades and releases proteoglycan with an associated reduction of sulphate incorporation. Similar cartilage from the femoral head responds to the retinol with an inhibition of sulphate incorporation but no degradation or loss of proteoglycan. Extraction of the proteoglycan from the femoral head samples failed to demonstrate any evidence of breakdown.

Key words: proteoglycan, chondrocyte, glycosaminoglycans.

Articular chondrocytes can be activated to degrade their surrounding matrix by factors released from other cells such as fibroblasts and mononuclear leucocytes. ${ }^{1-3}$ This has increased interest in the possible role of chondrocytes in the breakdown of cartilage in arthritis. There is evidence both in rheumatoid arthritis ${ }^{4}$ and in osteoarthritis ${ }^{6} 7$ that the chondrocytes are contributing to the breakdown of matrix, but the factors that control this function are unknown.

Clinical studies indicate that some joints are much more prone to arthritis than others. For example, the knee joint is very commonly involved in osteoarthritis, while the hip joint is less often affected. ${ }^{8}$ The reasons for this and other patterns of joint involvement are unknown.

Retinol (vitamin A) in pharmacological doses will activate articular chondrocytes to degrade the extracellular matrix. This effect has been demonstrated in cartilage from a variety of sources including chick limb bone rudiments, ${ }^{9}$ pig articular cartilage, ${ }^{10}$ and young adult human cartilage. ${ }^{11}$

The present work examines the response to retinol of chondrocytes in weight-bearing regions of human femoral head and femoral condyle cartilage. The effect of this catabolic agent on the breakdown of proteoglycan and the synthesis of new proteoglycan has been studied.

\footnotetext{
Accepted for publication 6 June 1984 .

Correspondence to Dr R. W. Jubb, Selly Oak Hospital, Raddlebarn Road, Birmingham.
}

\section{Materials and methods}

\section{MATERIALS}

Human femoral head cartilage. Femoral heads removed after fracture of the femoral neck were immediately placed into sterile Hanks solution containing penicillin and streptomycin. Fullthickness samples of cartilage, weighing $5-10 \mathrm{mg}$, were dissected from the superolateral weightbearing region of the femoral head. Samples were set up in culture within three hours of excision of the femoral head.

Human femoral condyle cartilage. A femoral condyle was removed from cadavers at necropsy within 24 hours of clinical death. All the patients had died suddenly of myocardial infarction, cerebrovascular accident, or trauma, and the joints were macroscopically normal. The condylar cartilage with underlying bone was placed in Hanks solution. Full-thickness samples of cartilage were removed from the central region of the condyle.

Bovine nasal cartilage. Small discs, $2 \mathrm{~mm}$ thick by $4 \mathrm{~mm}$ diameter, of bovine nasal cartilage were dissected within three hours of the animals being slaughtered. ${ }^{12}$

\section{METHODS}

Organ culture. Explants of cartilage were maintained in microtitre plates (Corning E11) with $250 \mu \mathrm{l}$ of culture medium for 12 days. The basic medium was Dulbecco's modification of Eagle's medium 
(DMEM) (Gibco-high glucose, no pyruvate) supplemented with ascorbic acid. ${ }^{1}$ In some cultures $10 \%$ heat inactivated normal rabbit serum or human serum was used. Retinol was used at $5 \times 10^{-6} \mathrm{M}$ or $1 \times 10^{-5} \mathrm{M}$. The culture medium was changed at three-day intervals with a final three-day pulse of ${ }^{35} \mathrm{SO}_{4}$ at $5 \mu \mathrm{Ci} / \mathrm{ml}$ (Amersham International). Samples were washed three times in phosphate buffered sulphate (same as phosphate buffered saline but with sulphate replacing the chloride). These samples were then frozen at $-20^{\circ} \mathrm{C}$ until required. The culture medium was also collected from each culture and stored at $-20^{\circ} \mathrm{C}$.

Extraction of ${ }^{35} S$ labelled glycosaminoglycans. The frozen explants were weighed and then dried at $37^{\circ} \mathrm{C}$ to constant weight. The dried sample was digested with papain (Sigma) and the glycosaminoglycan precipitated with $5 \%$ cetyl pyridinium chloride. Aliquots of both the precipitate (dissolved in formic acid) and the supernatant were counted on a B counter (Packard Tri-Carb 300) and expressed as disintegrations per minute (dpm).

Glycosaminoglycan assay. The glycosaminoglycans in the explant and the medium were assayed, after papain digestion, by their ability to bind to dimethylmethylene blue. ${ }^{13}$ Whale chondroitin sulphate (Koch-Light) was used as a standard.

Sulphate assay. The total inorganic sulphate in the culture medium was measured by precipitation with ${ }^{133}$ barium after uranyl acetate treatment to remove protein and phosphate. ${ }^{14}$

Extraction of proteoglycan. Proteoglycan was extracted from cartilage samples with $4 \mathrm{M}$ guanidinium chloride in $0.05 \mathrm{M}$ acetate buffer $\mathrm{pH} 5 \cdot 8.300$ $\mu \mathrm{l}$ was used for $10 \mathrm{mg}$ wet weight tissue. The extraction continued for 5 days at $4^{\circ} \mathrm{C}$. This procedure was found to extract $60-70 \%$ of the total ${ }^{35} \mathrm{~S}$. Enzyme inhibitors were added to the extraction fluid $(5 \mathrm{mM}$ phenylmethylsulphonylfluoride, $1 \mathrm{mM}$ 4-chloromercuribenzoate, $1 \mathrm{mM}$ 0-phenanthroline, and $1 \mu \mathrm{g} / \mathrm{ml}$ pepstatin). Fragments of human cartilage this size release proteoglycan monomer without the hyaluronic acid. ${ }^{15}$

Sepharose chromatography. $50 \mu \mathrm{l}$ of the extraction solution was applied to sepharose $2 \mathrm{~B}$ (Pharmacia) column $(0.6 \times 20 \mathrm{~cm})$ and eluted with $4 \mathrm{M}$ guanidinium chloride in $0.05 \mathrm{M}$ sodium acetate. The void and total volumes were indicated by ${ }^{35} \mathrm{SO}_{4}$ labelled proteoglycan aggregates from bovine nasal cartilage and free ${ }^{35} \mathrm{SO}_{4}$. Similar separations were performed with Sepharose 6B. Alternate 2-drop fractions were assayed for ${ }^{35} \mathrm{~S}$ activity or glycosaminoglycan by the dimethylmethylene blue (DMMB) assay.

Histology. Cartilage explants were fixed in $10 \%$ buffered formalin for 48 hours, dehydrated with ethanol, and embedded in glycolmethacrylate (Agar Aids). ${ }^{16} 2 \mu \mathrm{m}$ sections were cut, coated with autoradiographic stripping film (Kodak AR10), and exposed for two weeks. The autoradiograph was developed with ID-19 and stained with Carazzi haematoxylin. ${ }^{17}$ Other sections were stained with toluidine blue to assess the pattern of proteoglycan staining.

\section{Results}

RELEASE OF PROTEOGLYCAN

Femoral head. Retinol at both $10^{-5} \mathrm{M}$ and $5 \times 10^{-6} \mathrm{M}$ had no detectable effect on the release of proteoglycan as measured by the DMMB assay (Table 1). In all six experiments there was no increased loss of protoglycan above the control. In two experiments retinol at additional doses of $2 \times 10^{-5}$ and $4 \times 10^{-5} \mathrm{M}$ had no effect.

Femoral condyle. In the four experiments with femoral condyle cartilage two showed a considerable release of proteoglycan. This loss could be measured biochemically (Table 1 ) and was also seen histologically (Figs. 1A, B).

It was not clear why there was no response by the femoral head cartilage and only a partial response by the femoral condyle cartilage. The possibility of the culture conditions being unsuitable was examined with cartilage that had been shown in previously published work to respond to retinol in vitro.

Bovine nasal cartilage. This cartilage responds vigorously to the retinol in some circumstances. ${ }^{12} \mathrm{As}$ can be seen from Table 2, there was an almost complete loss of proteoglycan. Histological examination confirmed this result. The incorporation

Table 1 Release of glycosaminoglycans $(G A G)$ from human cartilage cultured for 12 days with $10 \%$ rabbit serum and retinol

\begin{tabular}{lcccc}
\hline $\begin{array}{l}\text { Source of } \\
\text { cartilage }\end{array}$ & & \multicolumn{2}{c}{$\%$ release of $G A G$} & \\
\cline { 2 - 5 } & & $\begin{array}{c}10^{-5} M \\
\text { retinol }\end{array}$ & $\begin{array}{c}5 \times 10^{-6} M \\
\text { retinol }\end{array}$ & Control \\
\hline Femoral head & $82 \mathrm{yr}$ & $3 \pm 1^{*}$ & $5 \pm 2$ & $4 \pm 2$ \\
Femoral head & $83 \mathrm{yr}$ & $11 \pm 5$ & $10 \pm 2$ & $11 \pm 3$ \\
Femoral head & $77 \mathrm{yr}$ & $5 \pm 1$ & $7 \pm 2$ & $5 \pm 1$ \\
Femoral head & $86 \mathrm{yr}$ & $5 \pm 2$ & $1 \pm 1$ & $1 \pm 1$ \\
Femoral head & $83 \mathrm{yr}$ & $8 \pm 3$ & $10 \pm 1$ & $8 \pm 1$ \\
Femoral head & $79 \mathrm{yr}$ & $7 \pm 2$ & $6 \pm 1$ & $5 \pm 1$ \\
& & & & $17 \pm 4$ \\
Femoral condyle & $76 \mathrm{yr}$ & $20 \pm 7$ & $15 \pm 3$ & $13 \pm 2$ \\
Femoral condyle & $80 \mathrm{yr}$ & $34 \pm 10$ & $20 \pm 6$ & $13 \pm 5$ \\
Femoral condyle & $83 \mathrm{yr}$ & $41 \pm 6$ & $32 \pm 6$ & $15 \pm 5$ \\
Femoral condyle & $82 \mathrm{yr}$ & $32 \pm 5$ & $30 \pm 8$ & $25 \pm 5$ \\
\hline
\end{tabular}

${ }^{*} \pm$ standard error of the mean. 


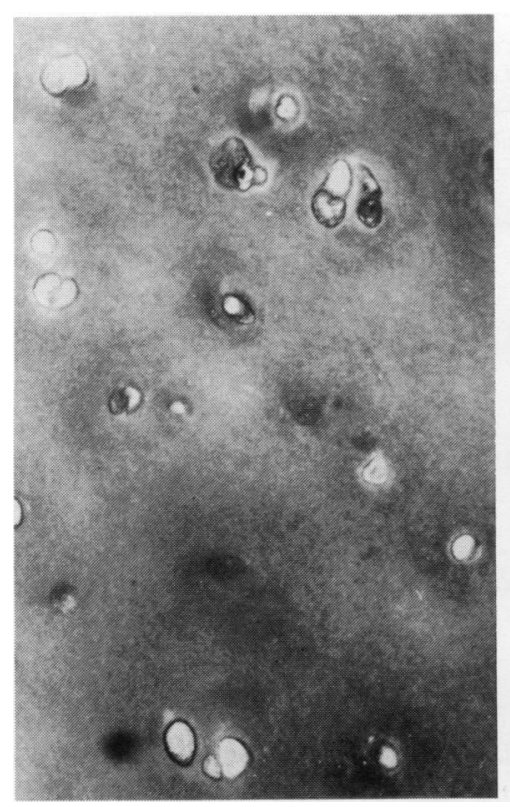

Fig. 1A

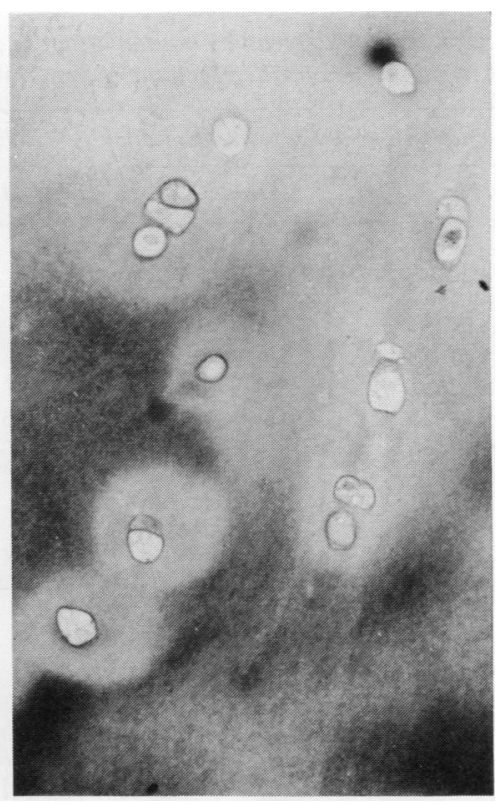

Fig. 1B
Fig. 1 A. Section of mid zone of femoral condyle cartilage after 12 days in DMEM+rabbit serum. $2 \mu$. (Toluidine blue, $\times 207$ ). B. Similar area as in $A$ but after culture in medium with $10^{-5} \mathrm{M}$ retinol. Note loss of proteoglycan staining. $2 \mu$ (Toluidine blue, $\times 207$ ). of sulphate into the glycosaminoglycan in the cartilage was markedly reduced. This could be inhibition of synthesis or rapid release from the cartilage.

Young human femoral condyle cartilage. Cartilage from this source aged $20-40$ yr has been shown to respond to retinol with loss of proteoglycan. ${ }^{11}$ In the system used in these experiments this cartilage also responds well to the retinol (Table 2) with a $60 \%$ release compared with $20 \%$ in the controls. Once again there was a reduction in the incorporation of ${ }^{35} \mathrm{SO}_{4}$ into the cartilage.

Table 2 Effect of retinol on the release of glycosaminoglycans $(G A G)$ and sulphation of proteoglycan by bovine nasal cartilage and young human articular cartilage cultured in $10 \%$ rabbit serum for 12 days

\begin{tabular}{lcll}
\hline & $\begin{array}{l}\text { dpm/mg wet } \\
\text { weight }\end{array}$ & $\begin{array}{l}\%{ }^{35} S \\
\text { inhibition }\end{array}$ & $\begin{array}{l}\% \text { release } \\
\text { of } G A G\end{array}$ \\
\hline Bovine nasal cartilage & & & \\
Control & $10008 \pm 5385^{*}$ & - & $34 \pm 10$ \\
$10^{-5} \mathrm{M}$ retinol & $504 \pm 164$ & 99 & $96 \pm 1$ \\
$5 \times 10^{-6} \mathrm{M}$ retinol & $549 \pm 294$ & 99 & $99 \pm 1$ \\
& & & \\
Young human cartilage & $4667 \pm 1236$ & - & $21 \pm 2$ \\
Control & $914 \pm 132$ & 81 & $63 \pm 6$ \\
$10^{-5} \mathrm{M}$ retinol & $1562 \pm 654$ & 67 & $58 \pm 9$ \\
$5 \times 10^{-6} \mathrm{M}$ retinol & & & \\
\hline
\end{tabular}

* \pm standard error of the mean.

\section{SULPHATION OF GLYCOSAMINOGLYCAN}

The lack of response of the chondrocytes in the femoral head cartilage could be due to poor viability of the cells, lack of penetration of retinol, toxicity of retinol, inability of cells to recognise retinol, or a difference in the metabolic responsiveness of the cells. Some of these aspects were resolved by examining the response of the cells in the cartilage to ${ }^{35} \mathrm{SO}_{4}$.

The chondrocytes in all samples studied were able to incorporate ${ }^{35} \mathrm{SO}_{4}$ into glycosaminoglycans. Killing the cells by freezing and thawing $(\times 2)$ abolished this effect (Table 3). By this criterion the chondrocytes were alive at the end of the 12-day culture period.

In the six experiments with femoral head cartilage there was an inhibition of incorporation of ${ }^{35} \mathrm{SO}_{4}$ into the glycosaminoglycans in the presence of retinol (Table 3 ). The mean inhibition was about $40-50 \%$. The femoral condyle cartilage showed a similar inhibition in all four samples studied (Table 3).

Autoradiographs of histological sections showed pericellular accumulation of radiolabel in all the control cartilage (Fig. 2). The reduction in radiolabelling with retinol was uniform throughout the cartilage. There was no obvious gradient in effect from the edge of the sample to the centre, nor was any zone of cartilage particularly affected. The histological appearance of the femoral head and 
Table 3 Effect of retinol on the sulphation of proteoglycan by human articular cartilage cultured in $10 \%$ rabbit serum for 12 days. Counts incorporated into cetyl pyridinium chloride precipitable material after papain digest

\begin{tabular}{|c|c|c|c|c|c|}
\hline \multirow[t]{2}{*}{ Source of cartilage } & & \multicolumn{2}{|c|}{$\mathrm{dpm} / \mathrm{mg}$ wet weight in control medium } & \multicolumn{2}{|c|}{$\%$ Inhibition of sulphation ${ }^{\dagger}$} \\
\hline & & Living & Dead & $10^{-5} \mathrm{M}$ retinol & $5 \times 10^{-6} M$ retinol \\
\hline Femoral head & $82 \mathrm{yr}$ & $940 \pm 110^{*}$ & - & $46 \%$ & $18 \%$ \\
\hline Femoral head & $83 y r$ & $370 \pm 210$ & $25 \pm 10$ & $69 \%$ & $49 \%$ \\
\hline Femoral head & $77 \mathrm{yr}$ & $555 \pm 140$ & $20 \pm 15$ & $27 \%$ & $12 \%$ \\
\hline Femoral head & $86 y r$ & $1433 \pm 1000$ & $29 \pm 12$ & $38 \%$ & $55 \%$ \\
\hline Femoral head & $79 \mathrm{yr}$ & $3476 \pm 2618$ & $240 \pm 41$ & $\begin{array}{l}68 \% \\
50 \pm 16\end{array}$ & $\begin{array}{l}37 \% \\
40 \pm 21\end{array}$ \\
\hline Femoral condyle & $76 \mathrm{yr}$ & $945 \pm 110$ & $20 \pm 16$ & $40 \%$ & $18 \%$ \\
\hline Femoral condyle & $80 \mathrm{yr}$ & $3154 \pm 1806$ & $65 \pm 49$ & $60 \%$ & $66 \%$ \\
\hline Femoral condyle & $83 y r$ & $4599 \pm 1555$ & $145 \pm 66$ & $82 \%$ & $74 \%$ \\
\hline Femoral condyle & $82 y r$ & $1876 \pm 1526$ & $192 \pm 129$ & $\begin{array}{l}46 \% \\
57 \pm 18\end{array}$ & $\begin{array}{l}73 \% \\
57 \pm 26\end{array}$ \\
\hline
\end{tabular}

* \pm standard error of the mean

${ }^{\dagger} 100$-test $\times 100 /$ living control.

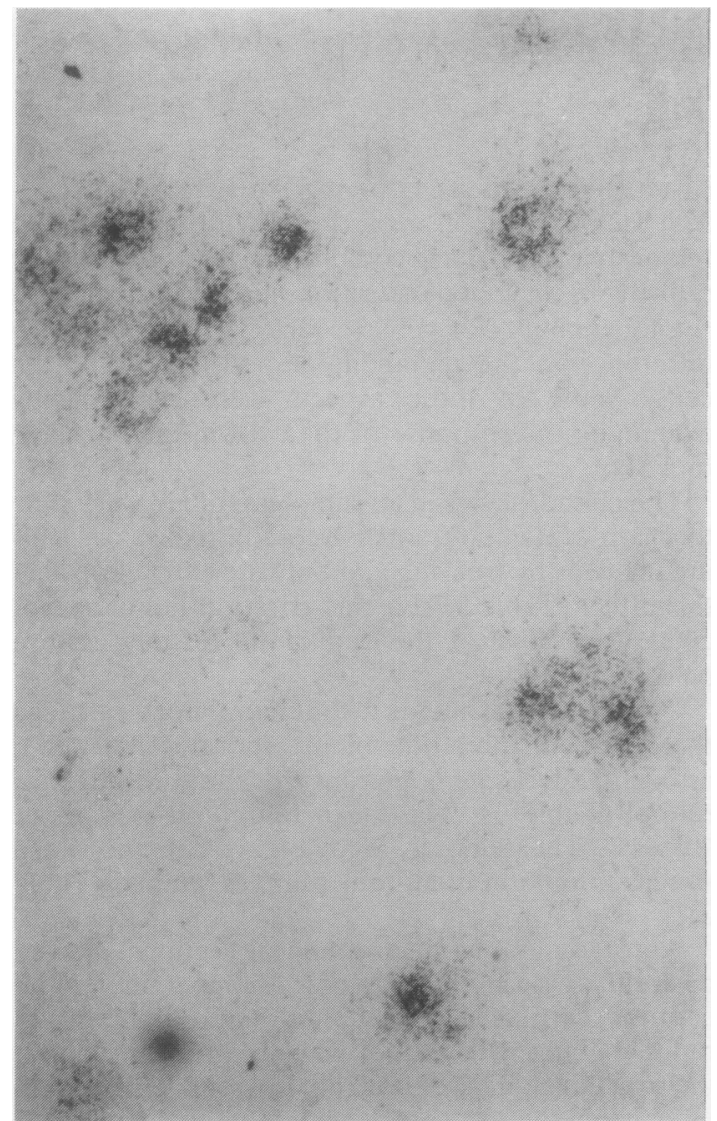

Fig. 2 Autoradiograph of section of mid zone of femoral head cartilage after 12 days in control medium with 5 $\mu \mathrm{Ci} / \mathrm{ml}^{35} \mathrm{SO}_{4}$ for final three days. (Unstained, $\times 300$ ). femoral condyle cartilage were similar, when stained either with toluidine blue or haematoxylin.

It is apparent, therefore, that the retinol reaches the femoral head chondrocytes and that they respond by a reduction in sulphation of glycosaminoglycan but not by a release of proteoglycan.

It was possible that the reduced sulphation indicated not a reduced synthesis but an increased? sulphatase activity. ${ }^{18}$ An attempt was made to assess this by measuring the total inorganic sulphate released into the culture medium. In two separate experiments there was no evidence of elevated sulphate in the medium (Table 4).

\section{RESPONSE TO RETINOL IN HUMAN SERUM}

It has previously been shown that chondrocytes in human articular cartilage have a different response to ascorbic acid when in human serum rather than bovine serum. ${ }^{19}$ In the present experiments the use of rabbit serum for the culture of human cartilage might account for the poor catabolic response to retinol. The experiments, therefore, were repeated with $10 \%$ human serum.

Table 4 Effect of retinol on the release of inorganic sulphate from femoral head cartilage in organ culture for 12 days

\begin{tabular}{llll}
\hline $\begin{array}{l}\text { Cartilage } \\
\text { sample }\end{array}$ & \multicolumn{2}{c}{ Concentration of inorganic sulphate $(\mathrm{mM})$} \\
\cline { 2 - 4 } & $10^{-5} M$ retinol & $5 \times 10^{-6} M$ retinol & Control \\
\hline $82 \mathrm{yr}$ & $0.96 \pm 0.03^{*}$ & $1.02 \pm 0.02$ & $1.03 \pm 0.05$ \\
$83 \mathrm{yr}$ & $1.02 \pm 0.03$ & $1.01 \pm 0.03$ & $0.95 \pm 0.06$ \\
\hline
\end{tabular}

* \pm standard error of the mean. 


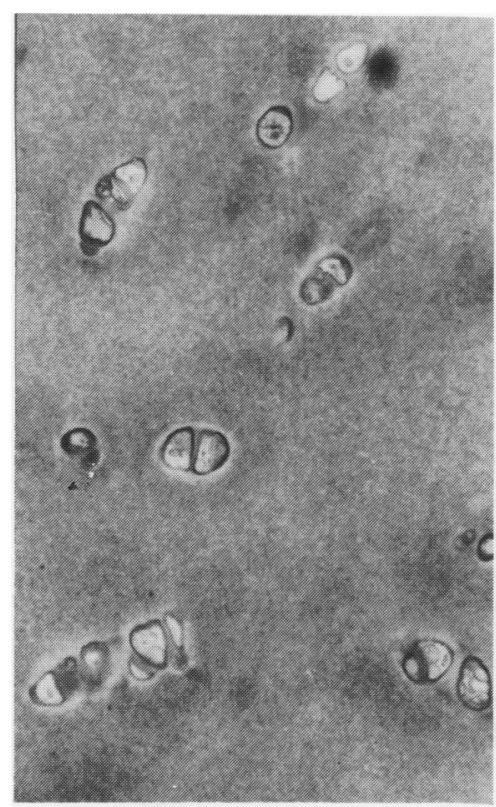

Fig. 3A

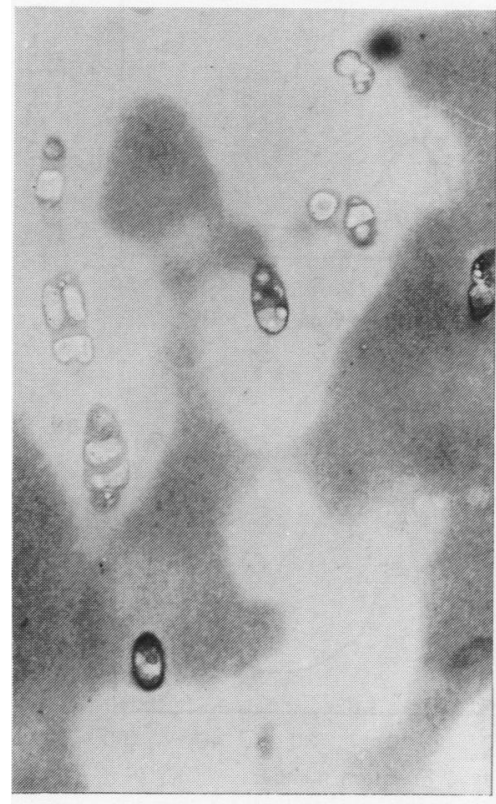

Fig. 3B

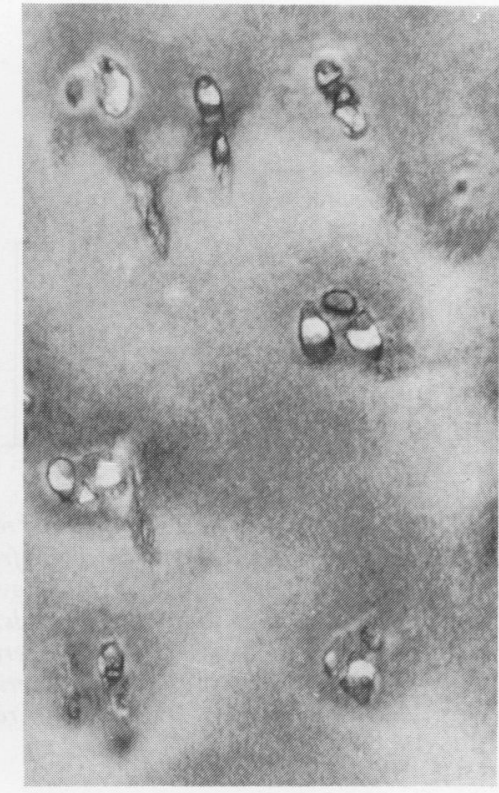

Fig. 3C

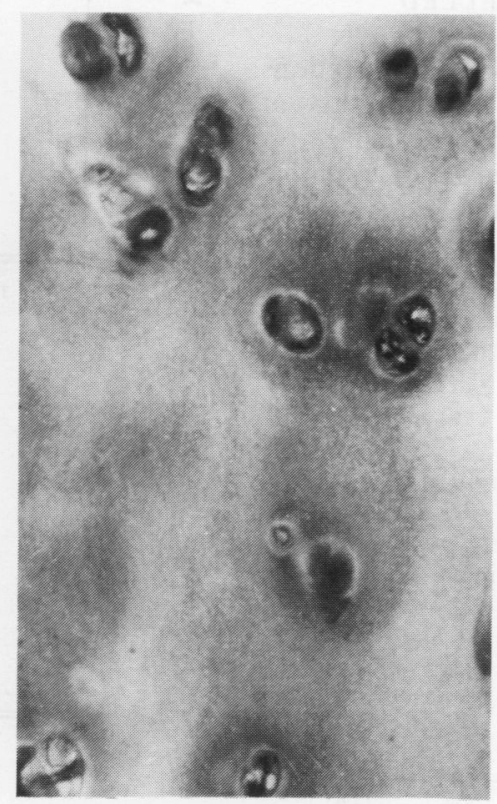

Fig. 3D
Fig. 3 A. Section of mid zone of femoral head cartilage after 12 davs in DMEM+human serum. (2 $\mu$ m thick, toluidine blue, $\times 207)$. B. Similar area as in $A$ but after culture in medium with $10^{-5} \mathrm{M}$ retinol. $(2 \mu \mathrm{m}$ thick, toluidine blue, $\times 207)$. C. Section of mid zone of femoral condyle cartilage after 12 days in DMEM+human serum. (2 $\mu \mathrm{m}$ thick, toluidine blue. $\times 207$ ). D. Similar area as in $C$ but after cultures in medium with $10^{-5} \mathrm{M}$ retinol. $(2$ m thick, toluidine blue, $\times 207)$.
Release of proteoglycan. With the substitution of the human serum all the samples of femoral condyle cartilage studied showed a clear response to the retinol with loss of proteoglycan (Table 5). This release was about $2-3$ times above the control levels
(Figs. 3A, B, C, D) The femoral head cartilage, however, did not show any significant response to the retinol (Table 5) (Fig. 3).

Sulphation of glycosaminoglycans. Once again the retinol produced a consistent reduction in the 
Table 5 Release of glycosaminoglycan $(G A G)$ from human cartilage cultured for 12 days with $10 \%$ human serum and retinol

\begin{tabular}{lcccc}
\hline $\begin{array}{l}\text { Source of } \\
\text { cartilage }\end{array}$ & \multicolumn{3}{c}{$\%$ release of $G A G$} \\
\cline { 2 - 5 } & & $\begin{array}{l}10^{-5} M \\
\text { retinol }\end{array}$ & $\begin{array}{l}5 \times 10^{-6} M \\
\text { retinol }\end{array}$ & Control \\
\hline Femoral head & $86 \mathrm{yr}$ & $6 \pm 1^{*}$ & $4 \pm 1$ & $3 \pm 1$ \\
Femoral head & $83 \mathrm{yr}$ & $11 \pm 2$ & $17 \pm 6$ & $12 \pm 4$ \\
Femoral head & $79 \mathrm{yr}$ & $9 \pm 2$ & $7 \pm 1$ & $6 \pm 1$ \\
Femoral condyle & $80 \mathrm{yr}$ & $47 \pm 3$ & $43 \pm 12$ & $14 \pm 3+$ \\
Femoral condyle & $83 \mathrm{yr}$ & $37 \pm 19$ & $30 \pm 12$ & $12 \pm 2++$ \\
Femoral condyle & $82 \mathrm{yr}$ & $51 \pm 10$ & $32 \pm 8$ & $23 \pm 4+++$ \\
\hline
\end{tabular}

* \pm standard error of the mean.

Retinol treatment compared with control. Analysis by paired $t$ test: $+=\mathrm{p}<0.0001,++=\mathrm{p}<0.03,+++=\mathrm{p}<0.005$.

sulphation of the glycosaminoglycans (Table 6). At least in the case of the femoral head cartilage, where there is no loss of proteoglycan this is likely to reflect a genuine inhibition of synthesis.

\section{EFFECT OF RETINOL ON PRELABELLED}

PROTEOGLYCAN IN FEMORAL HEAD

Although the experiments in the preceding sections have been unable to demonstrate any active loss of proteoglycan from the femoral head cartilage, it could be that the retinol was having a degradative effect but that the breakdown products were not being released into the medium.

To examine this possibility, femoral head cartilage was prelabelled with ${ }^{35} \mathrm{SO}_{4}$ to provide an additional method for monitoring the proteoglycan.

Table 6 Effect of retinol on the sulphation of proteoglycan by human articular cartilage cultured in $10 \%$ human serum for 12 days. Counts incorporated into cetyl pyridinium chloride precipitable material after pepsin digest

\begin{tabular}{|c|c|c|c|c|}
\hline \multirow[t]{2}{*}{$\begin{array}{l}\text { Source of } \\
\text { cartilage }\end{array}$} & & \multirow{2}{*}{$\begin{array}{l}\text { dpm/mg wet } \\
\text { weight in } \\
\text { control medium }\end{array}$} & \multicolumn{2}{|c|}{$\begin{array}{l}\% \text { Inhibition of }^{+} \\
\text {sulphation }^{+}\end{array}$} \\
\hline & & & $\begin{array}{l}10^{-5} \mathrm{M} \\
\text { retinol }\end{array}$ & $\begin{array}{l}5 \times 10^{-6} M \\
\text { retinol }\end{array}$ \\
\hline Femoral head & $86 y r$ & $1644 \pm 1582^{*}$ & $20 \%$ & $24 \%$ \\
\hline Femoral head & $83 \mathrm{yr}$ & $8279 \pm 3543$ & $58 \%$ & $41 \%$ \\
\hline Femoral head & $79 \mathrm{yr}$ & $11745 \pm 8467$ & $\begin{array}{l}77 \% \\
51 \pm 29\end{array}$ & $\begin{array}{l}73 \% \\
46 \pm 24\end{array}$ \\
\hline Femoral condyle & $80 y r$ & $15793 \pm 3260$ & $68 \%$ & $75 \%$ \\
\hline Femoral condyle & $83 \mathrm{yr}$ & $20574 \pm 2718$ & $78 \%$ & $80 \%$ \\
\hline Femoral condyle & $82 \mathrm{yr}$ & $18563 \pm 9068$ & $\begin{array}{l}76 \% \\
74 \pm 5\end{array}$ & $\begin{array}{l}70 \% \\
75 \pm 5\end{array}$ \\
\hline
\end{tabular}

* \pm standard error of the mean.

${ }^{\dagger} 100-$ test $\times 100$ living control).

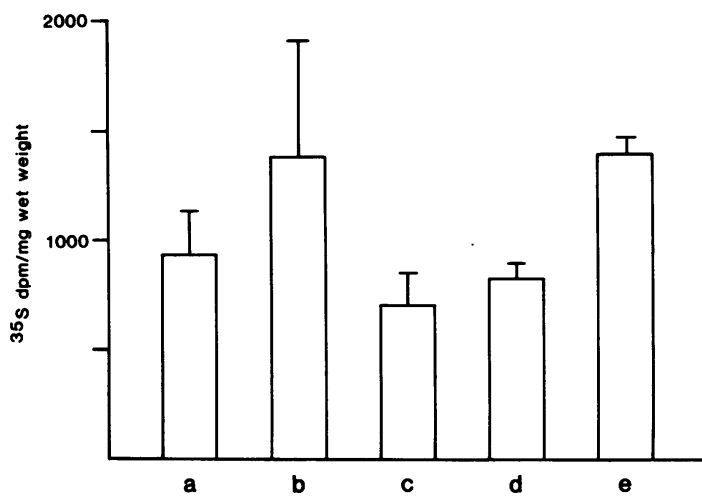

Fig. 4 Effect of retinol on the release of ${ }^{35} \mathrm{SO}_{4}$-associated macromolecules from femoral head cartilage prelabelled with ${ }^{35} \mathrm{SO}_{4}$. 12-day cultures with medium changed at three-day intervals, collected and dialysed. (a) $10^{-5} \mathrm{M}$ retinol + rabbit serum; (b) $5 \times 10^{-6} \mathrm{M}$ retinol + rabbit serum; (c) $10 \%$ rabbit serum; (d) DMEM alone; (e) dead cartilage in DMEM. Bars represent SEM.
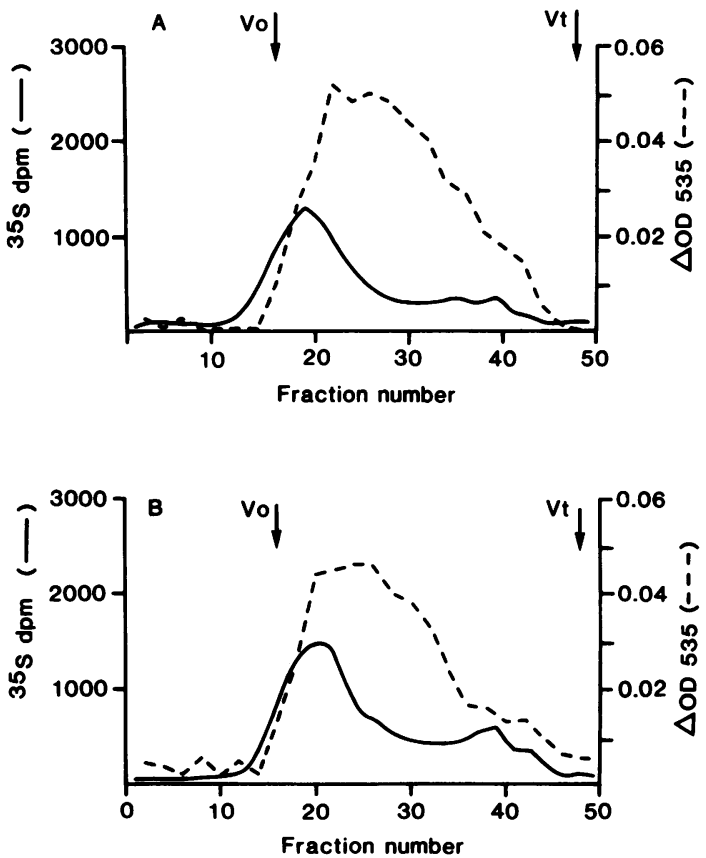

Fig. 5 Effect of retinol on the size of $4 M \mathrm{GnCl}$ extractable proteoglycan. Femoral head cartilage prelabelled with ${ }^{35} \mathrm{SO}_{4}$. Sepharose $2 \mathrm{~B}$ separation. $\mathrm{A}:-{ }^{35}$ S labelled proteoglycan; - _- glycosaminoglycans in proteoglycan from retinol treated cartilage.

B: $-{ }^{35}$ S labelled proteoglycan; - - - - glycosaminoglycan in proteoglycan from control cartilage. 


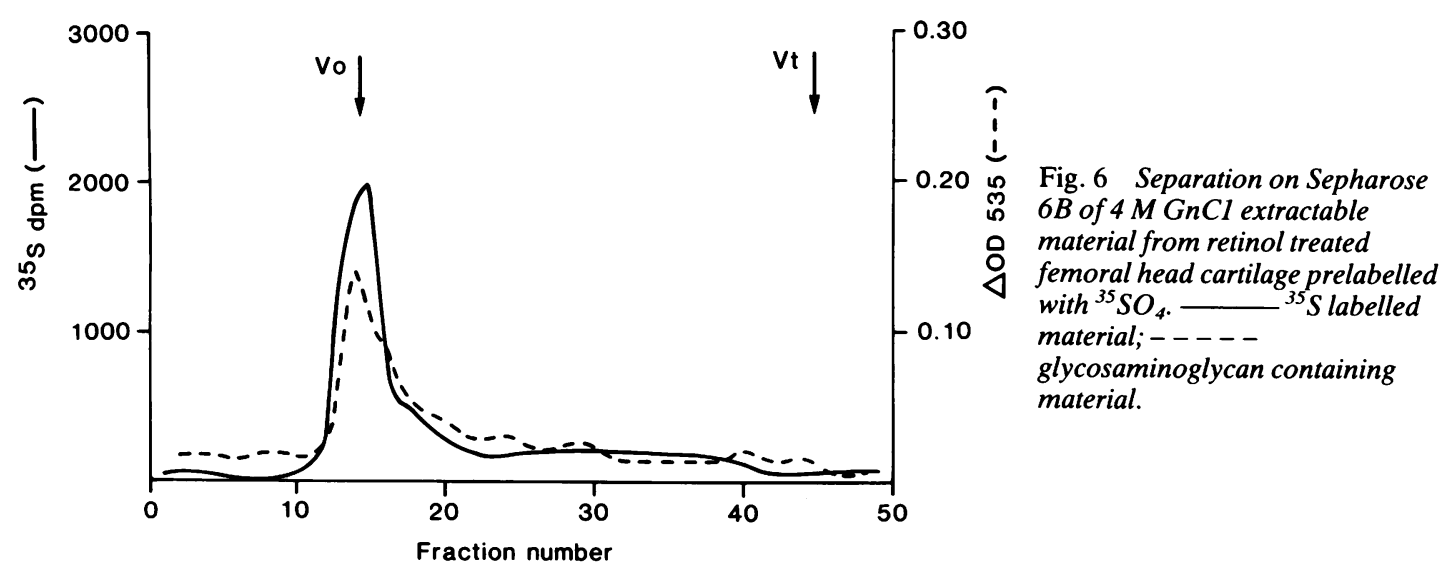

The release of ${ }^{35} \mathrm{SO}_{4}$-labelled non-dialysable material into the medium confirmed that the retinol did not cause any increased release of proteoglycan above the dead controls (Fig. 4).

The proteoglycan that could be extracted with 4 $\mathrm{M}$ guanidinium chloride was further analysed. Separation on a Sepharose 2B column failed to demonstrate any reduction in the size of the proteoglycan in the retinol treated group (Fig. 5). Both the labelled proteoglycan and the unlabelled material gave similar profiles in the control and the retinol group. It is not clear why the radiolabelled material is not the same as the total proteoglycan profile.

The presence of free glycosaminoglycans was examined by separation on a Sepharose 6B column (Fig. 6). There was no evidence from the elution profile to suggest the presence of breakdown fragments in the retinol treated group.

\section{Discussion}

The results presented show a qualitative difference between hip chondrocytes and knee chondrocytes from weight-bearing regions in their response to retinol. Despite retinol penetrating the cartilage and affecting both types of chondrocytes only those in the knee cartilage degrade the extracellular proteoglycan. It is quite possible that the hip chondrocytes could respond under different conditions. However, the results do demonstrate that, although the cartilages appear the same histologically, there are clear differences in their metabolic activity. This lack of proteoglycan breakdown could be due to the chondrocytes failing to produce the necessary degradative agents, the presence of antagonists, or the proteoglycan being more resistant to these agents.

Retinol is rapidly oxidised and inactivated if it is not bound to serum proteins in tissue culture medium. Rabbit serum was used in the present experiments, since it was entirely satisfactory for cultures of pig articular cartilage. ${ }^{10}$ However, the lack of response in the femoral head cartilage and the inconsistent response in the femoral condyles meant that the experiments could not be satisfactorily interpreted. It was found that substituting the rabbit serum with human serum gave consistent but different results in the two cartilage groups. Human serum differs in many ways from rabbit serum, and no attempt has been made in this paper to identify which factors might be relevant. Krystal et al. found that DNA synthesis was stimulated by ascorbate in human articular cartilage in $10 \%$ human serum but not in $10 \%$ fetal bovine serum. ${ }^{19}$

In experimental situations where the retinol stimulates considerable proteoglycan release it is not easy to be sure that any reduction in the incorporation of ${ }^{35} \mathrm{~S}$ reflects a direct inhibition of proteoglycan synthesis. However, with the femoral head cartilage, where there is no loss of proteoglycan, the retinol does inhibit the total incorporation of sulphate into proteoglycan. This reduction might be due to an inhibition of core protein synthesis, alteration of the glycosaminoglycan chain synthesis, or decreased sulphation. The biological significance of the observation is not clear, since retinol in sufficient dose will kill the cells. The importance, however, in the context of this paper is that it indicates that the retinol has reached the hip chondrocytes and that the cells have been able to respond to this potential catabolic agent.

Although the femoral head consistently looked normal and this source of cartilage is commonly used in studies of normal human cartilage, it should be remembered that the cartilage comes from patients who have fractured their necks of femur; 
many of these people have osteoporosis. It is not clear whether or not cartilage from such patients should be considered as normal. ${ }^{20}$ The younger femoral condyle cartilage responded much more to retinol than the very old cartilage, but there are not enough data to know whether there is an age related variation as well as a variation between joints. The experiment was performed with the younger tissue to check the experimental conditions and act as a positive control. ${ }^{11}$ A further study of the response of femoral head cartilage from different sources and ages is now required.

In chick limb bone rudiments retinol has been shown to degrade proteoglycan into fragments containing 4-6 polysaccharide chains. ${ }^{18}$ If this mechanism applied in the femoral head cartilage, then, even if the proteoglycan was not released, the fragments should be easily seen on the Sepharose separation. The prelabelled proteoglycan, being next to the chondrocyte, might also be expected to be sensitive to a minor degradative action of retinol. The results, however, clearly show that there has been no breakdown of the unlabelled or labelled proteoglycan in the hip cartilage. These results were further confirmed by the lack of release of sulphate into the medium. Based on previously published data $^{21}$ the potential release of sulphate from the cartilage could be about four-fold the concentration in the medium.

It is of interest that the hip cartilage used in these experiments is peculiarly resistant to the degradative effects of retinol. The hip joint is often spared in generalised osteoarthritis, ${ }^{8}$ and patients with osteoporosis are often spared osteoarthritis. The results of the experiments reported here provide a possible explanation, since, if the hip and the knee cartilage were exposed to a catabolic factor in vivo, and they responded as suggested by the experiments, then the knee cartilage would suffer much more. Such a direct extrapolation of the data is not justified with our present meagre knowledge of the metabolic state of the chondrocytes in different joints in humans. At present we do not know if the mechanism of action of the retinol is in any way similar to that of the possible physiological factors. The results, however, do indicate an in-vitro difference in metabolic reactivity which requires further study. It is important to establish whether or not this is due to resistance of the matrix to the degradative process or a different response of the chondrocytes to the retinol, and also to ascertain if it is unique to the particular source of cartilage used in this study. Further investigations are in progress.

I am indebted to Miss Joanna Hewitt for expert technical assistance and to $\mathrm{Mr} \mathrm{C}$. Green for the art work and photographic plates. I am grateful to the staff of the orthopaedic theatre and the post-mortem room at Addenbrooke's Hospital, Cambridge. for providing the human cartilage. I thank Dr J. T. Dingle for his support and encouragement and the Medical Research Council for the project grant support.

\section{References}

1 Fell H B. Jubb R W. The effect of synovial tissue on the breakdown of articular cartilage in organ culture. Arthritis Rheum 1977: 20: 1359-71.

2 Pilsworth L M C. Saklatvala J. The cartilage-resorbing protein catabolin is made by synovial fibroblasts and its production is increased by phorbol myristate acetate. Biochem J 1983; 216: 481-9.

3 Saklatvala J, Sarsfield S J. Lymphocytes induce resorption of cartilage by producing catabolin. Biochem $J$ 1982: 202: 275-8.

4 Muirden K D. Deutschmann P. Phillips M. Articular cartilage in rheumatoid arthritis: ultrastructure and enzymology. $J$ Rheumatol 1974: 1: 24-33.

5 Mitchell N S. Shepard N. Changes in proteoglycan and collagen in cartilage in rheumatoid arthritis. J. Bone Joint Surg 1978: 60A: $349-54$

6 Ali S Y. Evans L. Enzymatic degradation of cartilage in osteoarthritis. Fed Proc 1973; 32: 1494-8.

7 Ehrlich M G. Mankin H J, Jones H, Wright R. Crispin C. Vigliani $C$. Collagenase and collagenase inhibitors in osteoarthritis and normal human cartilage. J Clin Invest 1977: 59: 226-33.

8 Kellgren $\mathrm{J} \mathrm{H}$. Osteoarthritis in patients and populations. $\mathrm{Br}$ Med J 1961; ii: $1-6$.

9 Fell H B, Mellanby E. The effect of hypervitaminosis A on embryonic limb bones cultured in vitro. $J$ Physiol (Lond) 1952; 116: $320-49$.

10 Jubb $\mathrm{R}$ W. Fell H B. The breakdown of collagen by chondrocytes. J Pathol 1980; 130: 159-67.

11 Saklatvala J, Sarsfield S J. Pilsworth L M C. Characterization of proteins from human synovium and mononuclear leucocytes that induce resorption of cartilage proteoglycan in vitro. Biochem J 1983; 209: 337-44.

12 Dingle J T. Saklatvala J, Hembry R. Tyler J. Fell H B, Jubb $\mathrm{R}$ W. A cartilage catabolic factor from synovium. Biochem $J$ 1979; 184: 177-80.

13 Farndale R W. Sayers C A. Barrett A J. A direct spectrophotometric microassay for sulphated glycosaminoglycans in cartilage cultures. Connect Tissue Res 1982; 9: 247-8.

14 Miller E, Hlad C J, Levine S, Holmes J H, Elrick H. The use of radioisotopes to measure body fluid constituents. 1. Plasma sulphate. J Lab Clin Med 1961; 58: 656-61.

15 Bayliss M J. Venn M. Maroudas A. Ali S Y. Structure of proteoglycans from different layers of human articular cartilage. Biochem J 1983; 209: 387-400.

16 Ruddell C L. Embedding media for 1-2 micron sectioning. 2. Hydroxyethyl methacrylate combined with 2-butoxyethanol. Stain Technol 1967: 42: 253-5.

17 Jubb R W. Fell H B. The effect of synovial tissues on the synthesis of protcoglycan by the articular cartilage of young pigs. Arthritis Rheum 1980; 23: 545-55.

18 Morrison R I G. The breakdown of proteoglycans by lysosomal enzymes and its specific inhibition by an antiserum to cathepsin D. In: Balazs E A, ed. The chemistry and molecular biology of the intercellular matrix. New York: Academic Press. 1970; 3: 1683-708.

19 Krystal G. Morris G M. Sokoloff L. Stimulation of DNA synthesis by ascorbate in cultures of articular chondrocytes. Arthritis Rheum 1982; 25: 318-25.

20 Aitken J M. Relevance of osteoporosis in women with fracture of the femoral neck. Br Med J 1983; 288: 597-601.

21 Maroudas A. Physicochemical properties of articular cartilage. In: Freeman M A R, ed. Adult articular cartilage. London: Pitman, 1979: 215-290. 